\title{
Évaluation de la technique de sexage et de foetométrie par échographie chez la chèvre du sahel
}

\author{
Moussa ZONGO ${ }^{1 *}$, Maria Teresa PELLICER-RUBIO ${ }^{2}$, Hamidou BOLY $^{3}$, \\ Mohammed Mahmoud ABABNEH ${ }^{4}$, Drissa SANOU ${ }^{1}$, \\ Raymond BELEMTOUGRI ${ }^{1}$ et Laya SAWADOGO ${ }^{1}$ \\ ${ }^{1}$ Laboratoire de physiologie animale, UFR/SVT, Université de Ouagadougou, \\ 03 BP 7021 Ouagadougou 03 - Burkina Faso. \\ ${ }^{2}$ Institut National de la Recherche Agronomique (INRA), Unité Mixte de Recherche(UMR) Physiologie de la \\ Reproduction et des Comportements (PRC). Centre de Tours. F-37380 Nouzilly, France. \\ ${ }^{3}$ Institut du Développement Rural, Université Polytechnique de Bobo-dioulasso, \\ 01 B.P. 1091 Bobo-Dioulasso 01, Burkina Faso. \\ ${ }^{4}$ Department of Veterinary Clinical Sciences, Faculty of Veterinary Medicine, \\ Jordan University of Science and Technology, Irbid, Jordan. \\ *Auteur correspondant, E-mail: moussa_zongo59@yahoo.fr ; moussa_zongo@univ-ouaga.bf ; \\ Tel: (+226)70-25-90-47; Fax: (226)50-30-72-42.
}

\section{RESUME}

Le succès de l'application de l'échographie en production animale est en grande partie lié à sa sensibilité et sa spécificité au diagnostic. Les protocoles de validation de la technique, bien que nombreux n'ont pas encore été appliqués aux organes génitaux après abattage. Cette étude vise à évaluer la technique pour la détermination du sexe et la foetométrie à partir d'utérus gravides collectés après abattage. Pour cela, les résultats échographiques de détermination du sexe $(n=52)$ et de mesures de la longueur du dos $(n=44)$ ont été comparés aux observations faites après dissection des utérus. L'évaluation de la technique a été réalisée par le calcul de la valeur prédictive et de la sensibilité. Les données ont été analysées au moyen du test de corrélation de Pearson et par régression linéaire. Les valeurs prédictives ont été de 69,23\% et de 30,76\% pour les diagnostics des sexes mâle et femelle, respectivement. La sensibilité a été de $75 \%$. L'équation de régression a été de LDO $=1,056 \mathrm{LDE}-0,450(\mathrm{r}=0,936, \mathrm{p}=0,008)$ (LDO et LDE en millimètre). La technique de l'échographie est valide pour la détermination du sexe et les mesures de longueur de dos chez la chèvre sahélienne.

(C) 2014 International Formulae Group. All rights reserved.

Mots clés: chèvre, échographie, fœtus, sensibilité, sexe.

\section{INTRODUCTION}

Ces dernières années, l'application de l'échographie à la reproduction des animaux domestiques a permis de générer d'importantes informations sur la dynamique des structures ovariennes, l'involution utérine et le développement du fœetus (Lee et al., 2005; Ali et Hayder, 2007). Le plus grand avantage de la méthode échographique réside dans son caractère non 
invasif, comparativement à la laparoscopie ou à la laparotomie. Cette technique n'est pas stressante et ne nécessite pas d'administration d'agents anesthésiques ou sédatifs. Elle offre en outre, la possibilité de réaliser des examens répétés sans affecter les potentialités de reproduction de l'animal ou causer des dommages au fœtus (Zambelli et al., 2002). L'échographie permet de déterminer le sexe $\mathrm{du}$ fœetus par localisation du tubercule génital, des testicules ou de la glande mammaire. Elle permet en outre, de déterminer le stade de la gestation et de prédire le poids du fœus ou du nouveau-né (Ali et Hayder, 2007 ; Vural et al., 2008). Dans les élevages tropicaux où les mâles et les femelles sont conduits ensembles, cette technique est un atout important pour une gestion optimale de la reproduction. Elle fournit aux producteurs, les informations nécessaires pour regrouper les femelles gestantes selon leur besoin nutritionnel et organiser un rationnement approprié au cours du dernier trimestre de la gestation, réaliser le tarissement à des périodes adéquates et les préparer à la parturition (Vural et al., 2008).

Dans la dynamique de la validation de la technique de l'échographie, différents protocoles ont été développés (Vinoles et al., 2004). Des essais d'observation de la présence des follicules préovulatoires et de détermination $\mathrm{du}$ moment et $\mathrm{du}$ taux d'ovulation ont été réalisés sur des chèvres super-ovulées (Menchaca et al., 2001). Récemment, l'échographie a été appliquée à la morphométries des structures ovariennes chez la brebis (Vinoles et al., 2004) et à la détermination de la taille, du nombre de follicules et de corps jaune (Simoes et al., 2005), du moment de l'ovulation (Baril et al., 2000) et du nombre de corps jaune pendant le cycle œstral chez la chèvre (Bouttier et al., 2000). Dans la majeure partie de ces études, l'évaluation de la technique a été réalisée comparativement à des méthodes de référence telles que la laparoscopie (Baril et al., 2000 ; Bouttier et al., 2000), la laparotomie (Menchaca et al., 2001), la dissection ovarienne ou les coupes histologiques (Simoes et al., 2005).

L'objectif de la présente étude a été d'évaluer l'application de la technique échographique pour la détermination du sexe et pour les mesures de longueur du dos chez la chèvre du sahel. A notre connaissance, cette étude est la première expérience à évaluer la technique de la détermination du sexe et de la fœtométrie par échographie sur des utérus gravides de chèvre collectés à l'abattoir. Il s'est agi d'apprécier la valeur prédictive, la sensibilité et la corrélation de la méthode échographique.

\section{MATERIEL ET METHODES Animaux}

La présente étude a concerné 52 utérus gravides de chèvres sahéliennes collectés après abattage. Le poids, l'âge et la parité des animaux à l'abattage ont été respectivement compris entre 35 et $40 \mathrm{~kg}, 30$ et 48 mois et 2 et 4 lactations. Les poids des fœtus ont été compris entre 2 et 600 grammes pour la foetométrie $(n=44)$ et 2 et 1987 grammes $(n=52)$ pour la détermination du sexe. Toutes les gestations étaient simples.

Les examens ont été réalisés par un même opérateur au moyen d'un échographe (Chison Medical Imagin co.ltd, 8300) équipé d'une sonde linéaire de $5 \mathrm{Mhz}$. Chaque appareil génital a été entièrement plongé dans une bassine d'eau selon la méthode décrite par Singh et al. (2004). La sonde, tenue de la main par le technicien est immergée dans l'eau et orientée en direction de l'utérus. L'opérateur effectue des mouvements de rotation autour de la corne gravide afin de visualiser les différents organes du fœetus. Le pouvoir de résolution de la sonde est de 2 millimètres. Les images vidéo des structures fœtales sont projetées sur l'écran. La détermination du sexe a consisté à l'examen des apparences du tubercule génital (GT) autour du cordon ombilical ou du scrotum ou de la glande mammaire entre les membres postérieurs selon la technique décrite par (Coubrough et Castell, 1998). Le fotus a été classé de sexe 
mâle quand le tubercule génital est localisé en position caudale immédiatement après l'attache abdominale du cordon ombilicale et/ou quand le scrotum a été observé entre les membres postérieurs (Figure 1B). Chez le fœtus femelle, le tubercule génital est situé vers la base de la queue et/ou la glande mammaire est présente entre les cuisses (Figure 1A) (Ali et Fahmy, 2008).

La longueur du dos du foetus a été déterminée (LDE) à l'aide d'instrument métrique intégré à l'appareil.

L'utérus a ensuite été disséqué pour mesurer la longueur du dos (LDO en millimètre) à l'aide de ruban métrique, déterminer le sexe à l'observation et peser le fœtus au moyen d'une balance. Les résultats échographiques ont été confrontés aux observations faites après dissection des utérus. La longueur du dos a été définie comme la distance entre l'éminence de la nuque et l'articulation du sacrum-coccygène comme décrit par Nwaogu et al. (2009).

\section{Analyses statistiques}

Dans la présente étude, la technique échographique a été évaluée par le calcul de la valeur prédictive et de la sensibilité (Pieterse et al., 1990 ; Vinoles et al., 2004).

La valeur prédictive a été définie comme la probabilité pour qu'une détermination du sexe mâle ou femelle soit correcte. La sensibilité mesure l'habileté du test à faire une détermination correcte du sexe (Vinoles et al., 2004). Les données collectées ont été rangées dans un tableau de contingence $2 \times 2$ pour la confrontation des résultats échographiques et anatomiques (Tableau 1).

La valeur prédictive $(100 \mathrm{a} /(\mathrm{a}+\mathrm{b}))$ et la sensibilité $(100 \mathrm{a} /(\mathrm{a}+\mathrm{c})$ ont été calculées à partir du nombre de détermination positive correcte de sexe mâle (a), du nombre détermination positive correcte de sexe femelle (b), du nombre de fausse détermination négative de sexe mâle (c) et du nombre de fausse détermination négative de sexe femelle (d) (Vinoles et al., 2004). Une corrélation a en outre été établie entre les mesures échographiques et anatomiques de la longueur du dos au moyen des tests de corrélation de Pearson et de régression linéaire du logiciel Excel 2010, avec les données échographiques comme variable dépendant. La courbe de tendance retenue est celle qui présente une forte corrélation significative optimale entre les deux lots de données.

\section{RESULTATS}

Le Tableau 2 présente l'analyse comparative des données sur le diagnostic du sexe obtenu à l'échographie avec ceux obtenus après dissection des utérus $(\mathrm{n}=52)$. Les valeurs prédictives et de sensibilité y sont également rapportées. Dans la présente étude, les valeurs prédictives de la technique échographique pour la détermination des sexes mâle et femelle sont respectivement de $69,23 \%$ et $30,76 \%$. La sensibilité totale de la technique échographique pour la détermination échographie du sexe de foetus de chèvre sahélienne est de 75\% (39/52). Cette sensibilité varie en fonction du sexe du foetus. Elle est de $80 \%$ (12/15) pour la détermination du sexe femelle et de $73 \%$ (27/37) pour la détermination du sexe mâle.

La Figure 2 rapporte la corrélation entre la LDE et la LDO des fotus de la chèvre sahélienne ( $\mathrm{n}=44)$.

L'équation de régression linéaire décrivant la courbe de tendance des nuages de points est : $y=1,056 x-0,450$, où y représente la mesure de la LDO (en millimètre) et $\mathrm{x}$ représente la variable dépendante LDE du fotus (millimètre). Il existe une corrélation hautement positive et significative $(r=0,961$; $\mathrm{p}=0,008)$ entre la LDO et la LDE. La dispersion des nuages de points autour de la droite de régression varie en fonction du poids $\mathrm{du}$ fotus (Figure 2). Les points sont plus regroupés autour de la droite de régression en début de gestation. 
Tableau 1: Tableau de comparaison des résultats échographiques et anatomiques de diagnostic du sexe.

\begin{tabular}{lccc}
\hline Résultats échographiques & \multicolumn{3}{c}{ Résultats anatomiques après dissection } \\
\cline { 2 - 4 } & Mâle & Femelle & Total \\
\hline Positif $(+)$ & $\mathrm{a}$ & $\mathrm{b}$ & $\mathrm{a}+\mathrm{b}$ \\
Négatif $(-)$ & $\mathrm{c}$ & $\mathrm{d}$ & $\mathrm{c}+\mathrm{d}$ \\
Total & $\mathrm{a}+\mathrm{c}$ & $\mathrm{b}+\mathrm{d}$ & $\mathrm{n}$ \\
\hline
\end{tabular}

Tableau 2 : Analyse comparée des résultats de diagnostic de sexe à l'échographie et après dissection $(\mathrm{n}=52)$, de la sensibilité $(\%)$ et de la valeur prédictive $(\%)$.

\begin{tabular}{lccc}
\hline Résultats échographiques & \multicolumn{3}{c}{ Résultats anatomiques après } \\
& dissection \\
\cline { 2 - 4 } & Mâle & Femelle & Total \\
\hline Positif (+) & 27 & 12 & 39 \\
Négatif (-) & 10 & 3 & 13 \\
Total & 37 & 15 & 52 \\
Valeur prédictive & $69,23 \%$ & $30,76 \%$ & \\
Sensibilité & $73 \%$ & $80 \%$ & $75 \%$ \\
\hline
\end{tabular}

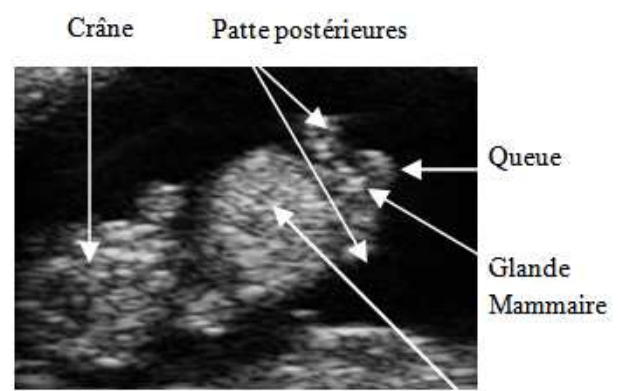

Cordon ombilical

(A) Fotus femelle

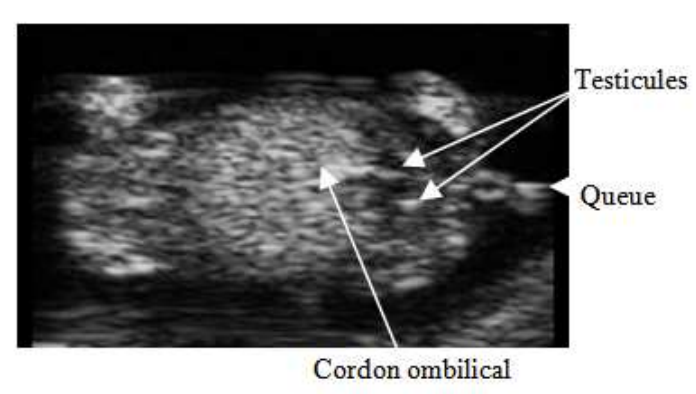

(B) Fotus mâle

Figure 1: Images échographiques d'un fœtus femelle (A) et d'un fœetus mâle (B) de chèvre Sahélienne. 


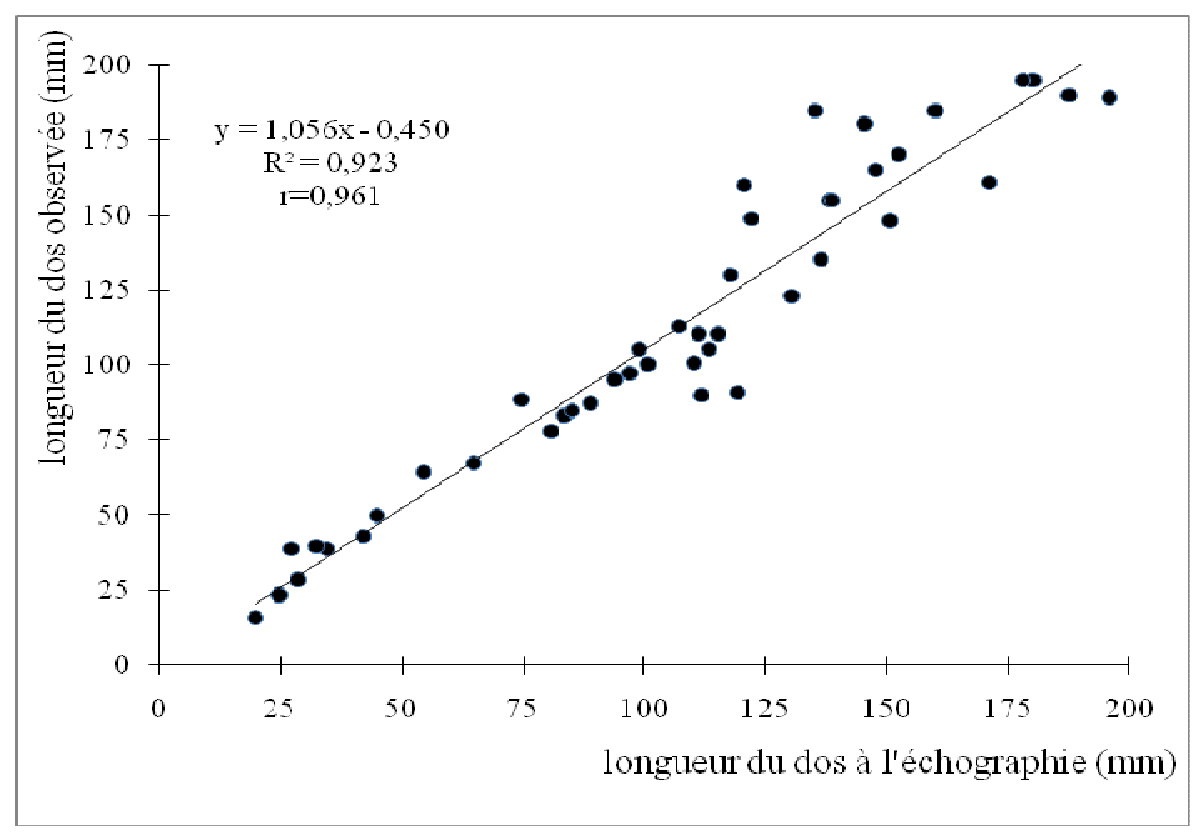

Figure 2 : Droite de régression linéaire montrant la relation entre LDE et la LDO. $(\mathrm{P}<0,01)$.

\section{DISCUSSION}

Dans la présente étude, la sensibilité de la technique de l'échographie pour la détermination du sexe de fotus a été de $75 \%$ (39/52). Ce résultat est compris dans l'intervalle de $[68,4 \%-83,3 \%]$ rapporté pour le sexage des portées simples et doubles chez la chèvre Baladi d'Egypte (Amer, 2007). Cette valeur est cependant en dessous de l'intervalle de [90\% - 100\%] rapporté par Santos et al. (2007) chez les races caprines toggenburg, Anglo-nubienne et Boers. Chez la brebis, la sensibilité de la technique a été de $89 \%$ avec des proportions de $100 \%$ et $77 \%$ pour la détermination des sexes mâle et femelle respectivement (Moraes et al., 2009). Chez la bufflesse, la sensibilité de l'échographie varie entre $64 \%$ et $97,1 \%$ selon les auteurs (Ali et Fahmy, 2008 ; Yotov et al., 2011 ). L'expérience de l'opérateur et le stade de développement du foetus au moment de l'examen expliquent en partie les résultats de notre expérience. En effet, la sensibilité et la valeur prédictive de l'échographie pour le sexage sont fortement liées à la migration et à la localisation finale du tubercule génital.
Chez la chèvre, la migration du tubercule génital commence à partir de 45 jours de gestation. La fenêtre de détermination du sexe se situe entre 55 et 70 jours afin de réduire les erreurs liées à l'opérateur et à l'espèce. (Santos et al., 2007).

Dans notre étude, la sensibilité de la technique échographique a été plus forte pour la détermination du sexe femelle que pour le sexe mâle. Cette observation a été rapportée chez la bufflesse avec des sensibilités de $85 \%$ et $33 \%$ respectivement, pour les déterminations des sexes femelle et mâle (Yotov et al., 2011). Cette différence est liée à la migration et à la localisation finale du tubercule génital. Chez le fœtus femelle, le parcours du tubercule génital vers sa position finale est court comparativement au mâle (Reichenbach et al., 2004). De plus la localisation des indices d'identification du sexe femelle a été aisément observable. Chez le mâle, l'image échographique des membres postérieurs ou du cordon ombilical interfère souvent avec l'image du tubercule génital, ce qui peut affecter la détermination du sexe. 
Cette difficulté a été rapportée par Santos et al. (2007).

Dans la présente étude, les valeurs prédictives pour la détermination des sexes mâle et femelle ont été respectivement $69,23 \%$ et $30,76 \%$. Les travaux sur la détermination de la valeur prédictive de la technique de l'échographie ont porté sur l'évaluation des dimensions et du nombre des structures ovariennes chez la brebis (Vinoles et al., 2004).

Habituellement, la précision de la technique échographique s'évalue par la valeur du coefficient de corrélation entre les mesures échographiques et les mesures anatomiques observées. Dans la présente étude, le coefficient de corrélation entre la LDE et la LDO a été hautement positif et fortement significatif $(r=0,961 ; p<0,01)$. Cette valeur est très proche des coefficients de corrélation de la longueur du fotus $(r=0,992)$ et des diamètres interne et externe $(r=0,972$; $\mathrm{r}=0,984) \mathrm{du}$ sac gestationnel de la chatte domestique (Zambelli et al., 2002). Chez la chèvre indienne par contre, les mesures de LDE ont été significativement plus élevées que les mesures de LDO avec un facteur de correction de 0,83 (Singh et al., 2004).

La courbe de tendance de la relation entre LDE et LDO a été une droite de régression linéaire d'équation $\mathrm{y}=1,056 \mathrm{x}$ 0,450 . Chez la chatte domestique, Zambelli et al. (2002) ont rapporté la même relation entre LDE et LDO avec une équation $\mathrm{y}=2,0087 \mathrm{x}$ 31,43 .

\section{Conclusion}

Les résultats de cette étude ont montré que la technique de l'échographie est appropriée pour la détermination du sexe et la fœtométrie chez la chèvre Sahélienne. Toutefois, afin de réduire le nombre de faux diagnostic lors de l'application de la technique en ferme, il est souhaitable de répéter les examens ou de les réaliser tard dans la gestation afin de pouvoir visualiser le tubercule génitale pendant sa localisation finale ou identifier les organes génitaux externes. Une fenêtre de détermination du sexe du fœtus au cours de la gestation reste à définir pour l'espèce afin d'optimiser les résultats et de stimuler l'adoption de la technologie.

\section{REMERCIEMENTS}

Ce travail a été possible grâce à l'appui technique et financier de l'Agence International pour l'Energie Atomique (AIEA, BKF 5008) / country program for Support development of small ruminants production in Burkina Faso, au Programme de Productivité Agricole en Afrique de l'Ouest (PPAAO)/ECOWARD/CORAF/FCN/Burkina Faso et à la Fondation Internationale pour la Science (FIS, Bourse B/3281-2, Dr Moussa ZONGO). Les auteurs voudraient exprimer toute leur gratitude à ces institutions pour leur accompagnement. Ils expriment toute leur reconnaissance à Mario PODESTA et Shamsudin Mohamed pour leurs conseils et leur foi en la recherche en Afrique. Il souhaite que les Professeurs Jean François Beckers, Christian Hanzen, Wéré PITALA et Noélita Melo De Sousa trouvent ici la sympathie de toute l'équipe du laboratoire de physiologie animale.

\section{REFERENCES}

Ali A, Fahmy S. 2008. Ultrasonographic fetometry and determination of fetal sex in buffaloes (Bubalus bubalis). Anim. Reprod. Sci., 106: 90-99.

Ali A, Hayder M. 2007. Ultrasonographic assessment of embryonic, fetal and placental development in ossimi sheep. Small Rumin. Res., 73: 277-282.

Amer HA. 2007. Determination of first pregnancy and fetal measurements in Egyptian Baladi goats. Veterinarski Italiana, 44: 429-437.

Baril G, Touze JL, Pignon R, Saumande J. 2000. Evaluation of the efficiency of transrectal ultrasound to study ovarian function in goats. Theriogenology, 53: 370 . 
Bouttier A, Pignon R, Touze JL, Furstoss V, Baril G. 2000. Détermination du moment optimum au cours du cycle sexuel pour dénombrer les corps jaunes par échographie transrectale chez la chèvre. 7èmes Rencontres Recherches Ruminants, Paris, 137p.

Coubrough CA, Castell MC. 1998. Fetal sex determination by ultrasonically locating the genital tubercule in ewes. Theriogenology, 50: 263-267.

Lee Y, Lee O, Cho J, Shin H, Choi Y, Shim W, Choi W, ShiN H, Lee D, Shin S. 2005. Ultrasonic measurement of fetal parameters for estimation of gestational age in Korean black goats. J. Vet. Med. Sci., 67: 497-502.

Menchaca A, Pinczack A, Rubianes E. 2001. Ultrasonography estimation of the ovulation rate and the length of the ovulation period in superovulated goats. Theriogenology, 55: 531.

Moraes EPBX, Freitas Neto LM, Aguiar Filho CR, Bezerra, FQG, Santos MHB, Neves JP, Lima PF, Oliveira MAL. 2009. Mortality determination and gender identification of conceptuses in pregnancies of Santa Ines ovine by ultrasound. Sth. Afr. J. Anim. Sci., 39(4): 307-312.

Nwaogu IC, Anya KO, Agada PC. 2010. Estimation of fetal age using ultrasonic measurements of different fetal parameters in red Sokoto goats (Capra hircus). Veterinarski Archiv., 80(2): 225233.

Pieterse MC, Taverne MAM, Kruip TAM, Willemse AH. 1990. Detection of corpora lutea and follicles in cows: A comparison of transvaginal ultrasonography and rectal palpation. Vet. Rec., 126(22): 552- 554.

Reichenbach HD, Santos MHB, Oliveira MAL, Meinecke-Tillmann S, Bürstel DM. 2004. Sexagem fetal na cabra e na ovelha por ultra-sonografia. In
Diagnóstico de Gestação na Cabra e na Ovelha, Santos MHB, Oliveira MAL, Lima PF (eds). Varela São Paulo: São Paulo; 117-136.

Santo MHB, Gonzalez CIM, Bezerra FQG, Neves JP, Reichenbach HD, Lima PF, Oliveira MAL. 2007. Sexing of Dorper sheep fetuses derived from natural mating and embryo transfer by ultrasonography, Reprod., Fert. Dev., 19: 366-369.

Simões J, Potes J, Azevedo J, Almeida JC, Fontes P, Baril G, Mascarenhas R. 2005. Morphometry of ovarian structures by transrectal ultrasonography in Serrana goats. Anim. Reprod. Sci., 85: 263-273.

Singh NS, Gawande PG, Mishra OP, Nema RK, Mishra UK, Singh M. 2004. Accuracy of ultrasonography in early pregnancy diagnosis in doe. Asian-Aust. J. Anim. Sci., 17(6): 760-768.

Viñoles C, Meikle A, Forsberg M. 2004. Accuracy of evaluation of ovarian structures by transrectal ultrasonography in ewes, Anim. Reprod. Sci., 80: 69-79.

Vural MR, Sel T, Karagul H, Ozenc E, Orman M, Izgur H, Kuplulu S. 2008. Ultrasonographic examinations of embryonic- fetal growth in pregnant Akkaraman ewes fed selenium supply and dietary selenium restriction. Rev. Méd. Vét., 159(12): 628-633.

Yotov S, Atanasov A, Geogiev P. 2011. Determination of fetal sex in Buffaloes through a single sonographic examination. Bulg. J. Vet. Med., 14(1): 39-44.

Zambelli D, Castagnetti C, Belluzzi S, Bassi S. 2002. Correlation between the age of the conceptus and various ultrasonographic measurements during the first 30 days of pregnancy in domestic cats (Feliscatus). Theriogenology, 57: 1981 - 1987. 